\title{
EL TELETRABAJO, LA UTILIDAD DIGITAL POR LA PANDEMIA DEL COVID-19*
}

\author{
TELEWORKING, THE DIGITAL UTILITY \\ DUE TO THE COVID-19 PANDEMIC
}

\author{
TÉLÉTRAVAIL, L'UTILITAIRE NUMÉRIQUE \\ DÛ̀ À LA PANDÉMIE COVID-19
}

Julio Ismael CAMACHO SOLÍS**

RESUMEN: La utilización de tecnología es ineludible en la organización del trabajo y el soporte técnico. En el actual contexto de la economía, la crisis financiera de las empresas y patrones, así como el grave efecto transversal de la pandemia del COVID-19, sectores e industrias estaban por lograr la modernidad tecnológica; no obstante, la virulencia del COVID-19 obligó a empleadores y empresas a diseñar un entorno laboral diferente. Hacia finales del siglo XX se estaban transformando las relaciones laborales de un mundo cambiante. Con ese panorama, los objetivos de esta colaboración son las de estimar, valorar, suponer y determinar qué actitudes, procedimientos, esquemas y relaciones laborales se adecuarán donde las prioridades tendrán que ser hacia el trabajo digital por el riesgo de quedar desfasadas, poniendo en peligro su existencia, por lo que se puede determinar la siguiente hipótesis: ¿es la contingencia sanitaria del COVID-19, así como los riesgos que implican para la salud personal, lo que adecuará las relaciones laborales y sociales de manera general?, ¿es entonces el escenario transformado de trabajo habitual en trabajo a distancia? Por ello se utilizará la metodología analítica y descriptiva para la integración de la información cualitativa, que describirá el análisis y síntesis de la información recopilada en fuentes fiables de investigación; así,

* Recibido el 15 de agosto de 2020 y aceptado para su publicación el 3 de septiembre de 2020.

** Profesor investigador en la Facultad de Contaduría y Administración Campus I de la Universidad Autónoma de Chiapas, México. Correo electrónico: academico.chiapas15@hotmail.com. 
el teletrabajo es la forma emergente que evoluciona y gradualmente aumenta en el mundo entero; en contrapartida, el trabajo presencial conlleva el riesgo generalizado que pone en riesgo la salud de los trabajadores, cambio que da lugar a una forma no común de trabajo en la que han sido factor determinante las tecnologías de la información y la comunicación.

Palabras claves: teletrabajo, pandemia, inserción.

ABSTRAC: The use of technology is inescapable, in the organization of work and in the technical support, in the current context of the economy, the financial crisis of companies and employers, as well as the serious cross-cutting effect of the pandemic of COVID-19, sectors and industries were yet to achieve technological modernity, the virulence of COVID-19, forces employers and companies to design a different working environment, towards the end of the twentieth century industrial relations were being transformed into the environment of a changing world, the objectives of this collaboration are to estimate, value, suppose, determine, that attitudes, procedures, schemes and industrial relations would be adapted where priorities will have to be towards digital work because of the risk of being outdated, endangering their existence, so that the following hypothesis can be determined is the health contingency of COVID-19, as well as the risks involved in personal health, which would suit the, as well as the risks involved in personal health, which would adapt labor and social relations in a general way? is then the transformed scenario of regular work into remote work? Therefore, the analytical and descriptive methodology will be used for the integration of qualitative information, which will describe the analysis and synthesis of information collected in reliable sources of research, teleworking is the emerging form that evolves and gradually increases worldwide, face-to-face work carries the widespread risk that puts workers' health at risk, this change gives rise to an uns common form of work in which it has been a factor determinant of information and communication technologies.

Keywords: Telework, Pandemic, Insertion.

RÉSUMÉ: L'utilisation de la technologie est incontournable, dans l'organisation du travail et du soutien technique, dans le contexte actuel de l'économie, la crise financière des entreprises et des employeurs, ainsi que l'effet transversal grave de la pandémie de 
COVID-19, les secteurs et les industries n'étaient pas encore à atteindre la modernité technologique, la virulence de COVID-19, oblige les employeurs et les entreprises à concevoir un environnement de travail différent, vers la fin du XXe siècle, les relations professionnelles se transformaient en un monde en mutation, les objectifs de cette collaboration sont d'estimer, de valoriser, de supposer, de déterminer, que les attitudes, les procédures, les schémas et les relations professionnelles seraient adaptés là où les priorités devront être envers le travail numérique en raison du risque d'être dépassées, mettant en danger leur existence, de sorte que l'hypothèse suivante puisse être déterminée est la contingence sanitaire de COVID-19, ainsi que les risques liés à la santé personnelle, qui ade l'adaptation des relations sociales et de travail d'une manière générale?, est-ce alors le scénario transformé du travail régulier en travail à distance? Par conséquent, la méthodologie analytique et descriptive sera utilisée pour l'intégration de l'information qualitative, qui décrira l'analyse et la synthèse de l'information recueillie dans des sources fiables de recherche, le télétravail est la forme émergente qui évolue et augmente progressivement dans le monde entier, le travail en face-à-face comporte le risque généralisé qui met la santé des travailleurs en danger, ce changement donne lieu à une forme commune de travail non commun dans lequel il a été un facteur déterminant des technologies de l'information et de la communication.

Mots-clés: télétravail, pandémie, insertion.

SumARIO: I. Origen, desarrollo, evolución. II. Teletrabajo y diversidad digital. III. Tecnoloia y tecnotrabajo. IV. Disrupción por teletrabajo. V. Marco jurídico en México. VI. Conclusiones. VII. Fuentes.

\section{ORIGEN, DESARROLLO, EVOLUCIÓN}

T a fuerza de trabajo o fuerza laboral es un término acuñado por Karl Marx en la obra El Capital, que representa el tratado del trabajo obrero más estudiado por los economistas, ahí se refiere a la capacidad física y mental, inherente a todo ser humano para realizar un trabajo, donde además los conceptos de trabajo y fuerza de trabajo no son sinónimos. Así, el trabajo es 
la materialización, la concreción del potencial representado por la segunda; el trabajo es entonces el resultado de emplear la fuerza de trabajo. ${ }^{1}$

La palabra fuerza proviene del vocablo latino fortia, que es sinónimo de esfuerzo, en tanto que la palabra trabajo tiene su origen etimológico también en latín trípaliare, concepto utilizado para definir un yugo con tres palos empleado para amarrar a los esclavos donde se les castigaba con azotes; la noción de fuerza de trabajo o fuerza, por lo tanto, está asociada a la habilidad tanto física como mental, propia de cada individuo, para desarrollar una cierta labor.

Lo que caracteriza la época capitalista es que la fuerza de trabajo asume, para el propio obrero, la forma de una mercancía que le pertenece, y su trabajo, por consiguiente, es la forma de trabajo asalariado, por ello el capitalismo es la forma superior y más compleja de la producción mercantil que se convierte en el modelo universal de producción basado en la división social del trabajo, en la propiedad privada y en la producción de mercancías para el mercado, para así satisfacer las disímiles necesidades del ser humano, sean de carácter material o espiritual.

El concepto de teletrabajo nace en Estados Unidos, en 1973, a partir de un grupo de científicos de la University of Southern California dirigidos por el físico Jack Nilles. Dicho grupo, en el marco de la crisis del petróleo, investigó la aplicación en el trabajo de las (entonces disponibles) tecnologías informáticas para reducir el traslado de trabajadores a las oficinas, remplazándolo por el traslado de la información necesaria para que el trabajador realice sus tareas desde su casa o centro de teletrabajo próximo a su domicilio. La hipótesis era que debía traer, aplicado a escala conveniente, reducciones del tránsito vehicular en horas pico y la consecuente reducción de emisión de gases dañinos para el medio ambiente. ${ }^{2}$

Como resultado de ese experimento se logró reducir la tasa de rotación de sus empleados y el resultado del programa fue un aumento del 18\% de la productividad de esos empleados, con una tasa de rotación reducida a cero y los costos de instalaciones mucho menor, se denominó Trade off Telecomunications Transportation, la cual dio nombre a la modalidad teletrabajo. ${ }^{3}$

1 Díaz, Viviana Laura, Teletrabajo y neurotecnología. Una guía imprescindible para gestionar el trabajo, Buenos Aires, Ediciones Granica, 2018, p. 158.

2 Rubbini, Nora Inés y Mancuso, G., “¿El teletrabajo necesita de una nueva metáfora?”, VI Jornadas de Sociología de la UNLP, Universidad Nacional de La Plata, Facultad de Humanidades y Ciencias de la Educación, La Plata, 2010, p. 56.

3 Ibidem, p. 57. 
Todas las mercancías son producto del trabajo, por lo consiguiente, la base del valor de cambio de las mercancías es el valor determinado por la cantidad de trabajo invertido en la producción de mercancías, donde el empleo de la técnica aplicada, la producción, la organización del trabajo y el expertiz.

Los tipos concretos de trabajo no sólo se caracterizan por la diversidad de los procedimientos, instrumentos y resultados del trabajo, sino generalmente por la diferencia en la preparación necesaria de los trabajadores. Los no calificados pueden ser realizados por todos los miembros de la sociedad aptos para el trabajo que tengan instrucción y el nivel cultural adecuado; otros tipos de trabajo, denominados calificados, necesitan una preparación especial previa. ${ }^{4}$

Son las empresas multinacionales, primero en Estados Unidos y luego en Europa, las que, en el contexto de deterioro del empleo formal, incorporaron el teletrabajo. A posteriori, el teletrabajo fue incorporado por los gobiernos como fuente de empleo (aunque precario) para los sectores desocupados.

Es preciso destacar que se tratan de al menos cuatro procesos históricos transformadores de las relaciones laborales. El primero evidenció marcadamente el paso de la producción manual a la mecanizada, entre 1760 y 1830 . El segundo, alrededor de 1850, introdujo la electricidad que permitió la manufactura en masa. El tercero, a mediados del siglo XX, protagonizó la llegada de la electrónica y las tecnologías de la información con las telecomunicaciones. Y el último, la llamada cuarta revolución industrial 4.0, trae consigo una tendencia a la automatización total de la manufactura con sistemas cibernéticos, el Internet de las cosas y la ya conocida nube digital. ${ }^{5}$

En el ámbito social, los mecanismos de regulación, control y representación instituyeron un tipo de contrato social de alcance nacional que humanizó las tendencias disolventes del capitalismo de libre competencia en el siglo XIX y que se extendió en el siglo XX. Se instala en Alemania con medidas de seguridad social introducidas por Bismarck a mediados del siglo XIX, después de la primera guerra mundial en Inglaterra con las leyes sociales Lord Beveridge y el New Deal de Roosevelt en Estados Unidos. El Estado benefactor está basado en cuatro principios: 1) derecho al trabajo, 2) lucha contra la pobreza, 3) protección frente a riesgos individuales y colectivos, y 4) promoción de igualdad de oportunidades.

4 Díaz, Viviana Laura, Teletrabajo y neurotecnología, cit., p. 159.

5 López-López, Pedro et al., Inclusión digital, un nuevo derecho bumano, Madrid, Educación y Biblioteca, 2009, p. 56. 
En el siglo XIX, la revolución industrial generó nuevas condiciones, así como diversos problemas que ninguno de los modelos sociales, económicos y políticos existentes podía resolver. ${ }^{6}$ El feudalismo, la monarquía y las religiones tradicionales no estaban preparadas para gestionar las metrópolis industriales, los millones de obreros desarraigados o la naturaleza siempre cambiante de la economía moderna.

\section{TELETRABAJO Y DIVERSIDAD DIGITAL}

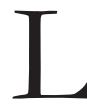

a crisis de COVID-19 ha determinado el experimento más grande a escala mundial para el teletrabajo. La pregunta central de esta colaboración es: cla crisis de COVID-19 nos ayudará a extender el teletrabajo como una formula normal/deseable para la organización de actividades de servicio y laborales?

En este complejo escenario, el teletrabajo se ha convertido en la dominante solución para mitigar el impacto del coronavirus tanto en el bienestar de la sociedad como en la actividad de los negocios; ha hecho que el teletrabajo pase de ser un privilegio a una necesidad. En consecuencia, muchas empresas han establecido protocolos diversos; algunas a marchas forzadas, acelerada improvisación, limitada conectividad, con la finalidad de que sus empleados puedan transitar de lo cotidiano a la conexión para el trabajo en forma digital.

A medida que se van consolidando los principios de la nueva revolución industrial que estamos viviendo desde finales del siglo XX y hasta nuestros días, empiezan a entreverse las tipologías de las nuevas formas de trabajar que tendrán mayoritariamente las empresas en el futuro y el perfil de trabajador apropiado para cubrir estas necesidades.

El teletrabajo ha planteado, ideológicamente, fuertes debates en torno a su conceptualización y ámbitos de estudio, lo importante es que su tratamiento permite optimizar la productividad del trabajador, gracias al aprovechamiento intensivo de su tiempo de trabajo. La reducción temporal y financiera del traslado de trabajadores implica un mejor aprovechamiento de la jornada de trabajo y una verdadera conciliación entre la vida familiar y laboral.

En 1990, la OIT definió teletrabajo como un trabajo efectuado en un lugar donde, apartado de las oficinas centrales o de los talleres de producción, el trabajador no mantiene contacto personal alguno con sus colegas, pero está

\footnotetext{
6 Yuval, Noah Harari, 21 lecciones para el siglo XXI, México, Editorial Debate, 2018, p. 54.
} 
en condiciones de comunicarse con ellos por medio de las nuevas tecnologías. Desde, 2005 esta misma organización lo hace de la siguiente manera: "Trabajo a distancia (incluido el trabajo a domicilio) efectuado con auxilio de medios de telecomunicación y/o de una computadora".?

El término teletrabajo está formado por el prefijo griego tele, que significa distancia o lejanía y que, en este caso, hace referencia a las telecomunicaciones. $\mathrm{Y}$ es que no es lo mismo trabajar desde casa que hacer teletrabajo, el prefijo tele ha sido parte de la evolución de las cosas usadas a la distancia como herramienta de utilidad o diversión, como telégrafo, teléfono, televisión, telefax, telescopio, telegrama, entre otros.

El teletrabajo es la forma de trabajo que se realiza en una ubicación alejada de una oficina central o instalaciones de producción, separando al trabajador del contacto personal con colegas de trabajo que estén en esa oficina. Las nuevas tecnologías de la información y la comunicación hacen posible esta separación facilitando la comunicación, lo que lo convierte en una realidad imparable para el escenario laboral que impera y con lo cual la pandemia del COVID-19 se mantendrá.

La telemática, que es una fusión de los términos telecomunicaciones e informática, se enfatiza como la disciplina científica y tecnológica que analiza e implementa servicios u aplicaciones que usan tanto los sistemas informáticos como las telecomunicaciones o cualquier tipo de comunicación por Internet.

El trabajo telemático se circunscribe al conjunto de actividades realizadas con el objetivo de alcanzar una meta, solucionar un problema, así como producir bienes y servicios para atender las necesidades humanas realizados a través de las tecnologías de la información y comunicación (TIC). Es decir, sin telecomunicaciones e informática no se da esta modalidad laboral, y sin el concepto de distancia tampoco cabe su aplicación. ${ }^{8}$

Por lo cual el teletrabajo es una especie dentro del trabajo a control remoto, ${ }^{9}$ que comparte sus mismas características generales que son:

a) se trata de una modalidad laboral;

b) se realiza en forma autónoma y asalariada;

c) se requiere la intermediación de las TIC, y

7 Tesauro OIT, http://wmw.ilo.org/public/libdoc/ILO-Thesaurus/spanish/, consultado el 23 de junio de 2020.

8 Castell, M., "Internet, libertad, y sociedad. Una perspectiva analítica", Polis, revista bolivariana, 2003, disponible en: http:/ / redalic.org/articulo.oa?, consultada el 28 de mayo de 2020.

9 Herrscher, E., Negocios, sociedad, trabajo y valores, Buenos Aires, 2013, p. 202. 
d) se ejecuta a distancia, en un lugar diferente del lugar de prestación de la tarea.

Diversidad de criterios reconocen al teletrabajo como un nuevo diseño espacial del proceso de producción, es una especie dentro del género, pero la tipología va más allá, pues se trata de una tarea a distancia, lo cual puede ser realizada en cualquier otro lugar diferente del establecimiento del empleador. Las TIC definen su naturaleza (vía telemática), y la existencia de subordinación lo determina como autónomo o dependiente; no obstante, está en discusión si su calidad de transversalidad le impide conformarse como la parte seccionada de un todo; por ello, el teletrabajo se inserta en la parte de un sector productivo que facilita la inclusión social y laboral de las personas mejorando su empleabilidad.

La OIT define el teletrabajo como una forma de organización del trabajo con las siguientes características:

- El trabajo se realiza en un lugar distinto del establecimiento principal del empleador o de las plantas de producción, de manera que el teletrabajador no mantiene un contacto personal con los demás trabajadores.

- Las nuevas tecnologías hacen posible esta distancia al facilitar la comunicación.

- El teletrabajo puede realizarse en línea, es decir, con una conexión informática, o fuera de línea.

- Puede organizarse de manera individual o colectiva, implicando la totalidad o una parte de las tareas del trabajador.

- Su implementación puede ser autónoma o como asalariado.

Con el teletrabajo en desarrollo que comprende tres generaciones; la primera se ubica en la oficina a domicilio, pues las computadoras y los teléfonos por su tamaño y conectividad permanecerían en la oficinas, lo que no permitía el trabajo nómada u movible, pasando por la oficina móvil y llegando a la oficina virtual; la segunda, la oficina móvil, rompió con la doble estructura espacial clásica del trabajo, ya que a partir de ese momento éste se podía hacer cada vez más fuera de los locales del empleador, en el domicilio del empleado pero también en otras localizaciones. ${ }^{10}$

10 Messenger, J. C. y Gschwind, L., "Telework and the Effects on Working", Time Life, Ginebra, OIT, 2015, p. 115. 
Con la generalización acelerada del Internet y el acceso a la red mundial surgió la actual generación del teletrabajo conocida como la oficina virtual, en la que se clasifican las tres generaciones del teletrabajo, como lo refiere Jessica Nicklin ${ }^{11}$ en el marco analítico ideal para definir el teletrabajo basado en:

1) La proporción del tiempo de trabajo puede teletrabajarse en parte o en toda la jornada laboral, lo cual determina dos subtipos:

a) teletrabajadores a tiempo parcial: desempeñan periódicamente las funciones del puesto fuera del centro principal de operaciones;

b) teletrabajadores a tiempo completo: normalmente desempeñan la mayoría o la totalidad de las funciones del puesto fuera del centro principal de operaciones.

2) Lugar de ejecución. Relacionado con dónde se cumple o ejecuta la tarea, también en este caso se reconocen dos subtipos:

a) fijo: el teletrabajador trabaja principalmente en un emplazamiento externo fijo, por ejemplo, en el domicilio;

b) móvil: el teletrabajador puede trabajar o trabaja en distintos lugares fuera del centro principal de operaciones.

3) Horario. Lo que en nuestra idiosincrasia seria jornada laboral; se reconocen dos subtipos:

a) fijo: los días/horas en que el trabajador desempeña sus funciones fuera del centro de operaciones son siempre los mismos;

b) variable: los días/horas en que el teletrabajador desempeñará sus funciones fuera del centro de operaciones varían.

4) Colaboración. Se refiere al teletrabajo en relación con el resto de los trabajadores presenciales, también se reconocen dos subtipos:

a) baja: cuando es limitada la interacción que los teletrabajadores han de mantener con los restantes trabajadores del centro principal de operaciones;

b) alta: cuando es importante la interacción que los teletrabajadores deben mantener con los trabajadores presenciales del centro principal de operaciones.

5) Sincronización de tareas. Referida al formato on-line/off-line, donde también se reconocen dos subtipos:

11 Nicklin, J. M., Telecommuting: What? Whit? When? and How?, Singanpur, Springer Science Media, 2016, p. 145. 
a) secuencial: cuando las tareas interdependientes del trabajador se desarrollan consecutivamente (por ejemplo, aplicaciones, correo electrónico);

b) simultánea: las tareas interdependientes del trabajador se desarrollan de forma simultánea (conferencia telefónica, videoconferencia, videollamada, foro virtual).

6) Autonomía. En referencia a poder optar por el teletrabajo, reconociéndose dos subtipos:

a) autonomía baja: los trabajadores tienen poco margen para decidir acogerse al teletrabajo;

b) autonomía alta: los trabajadores tienen mucho margen para decidirse acogerse al teletrabajo, así como cuándo y cómo lo hacen. ${ }^{12}$

La dificultad estriba en la falta de una definición generalmente aceptada de manera global y uniforme. Por un lado, la palabra es utilizada con significados ligeramente diferentes y, por otro, términos con significados muy similares al de teletrabajo son de uso común. La diversidad de definiciones se refleja que, sobre el tema, sólo de la conceptualización se puede comprobar que, además de teletrabajo, se utilizan habitualmente otros términos como teledesplazamiento (telecommuting), trabajo en red (networking), trabajo a distancia (remote working), trabajo flexible (flexible working), trabajo en el domicilio (bomeworking), trabajo virtual, trabajo remoto, trabajo distribuido, trabajo flexible, trabajo blando, trabajo adaptado, trabajo digital.

Se debe destacar en la ubicación de cada apreciación del concepto de teletrabajo tres criterios principales: el modo de organización, el lugar de ejecución y la tecnología utilizada, lo cual engloba la diversidad de entender cada definición existente hoy día, donde más del $60 \%$ se basa en una combinación de dos o más de dichos preceptos. Por ello, debido a la tecnología y el lugar donde se realizan las tareas, producen la creación de la siguiente tipología que complementa el teletrabajo como actividad cotidiana: a) escritorio multiusario, donde el teletrabajador realiza su tarea en forma mixta: a distancia y en la oficina principal; b) escritorio multiusuario con reserva, con reservación y notificando qué días van a asistir a la oficina principal; c) telecentros que son instalaciones donde se ofrecen estaciones y equipos de trabajo en diversos lugares; d) oficinas colaborativas para entorno de trabajo virtual donde los trabajadores y directivos realizan sus respectivas tareas de colaboración desde

12 Ibidem, p. 148. 
distintos lugares $;{ }^{13}$ e) teletrabajo móvil donde la conectividad se da a través durante su desplazamiento con teléfonos inteligentes de última generación; f) teletrabajo complementario, que se realiza en casa de los trabajadores tras la jornada laboral, fines de semana o días festivos, este modalidad de teletrabajo es equivalente a horas extras no remuneradas.

Este nuevo enfoque del trabajo implica, como vemos, una transferencia del control desde la empresa al empleado; en la era del trabajo digital e inteligente, los individuos son los dueños de la gestión de su tiempo y de su productividad.

Esta idea es importante porque el teletrabajo se puede llegar a confundir con otras actividades que se llevan a cabo mediante las nuevas tecnologías como telemarketing, teleservicios o televenta, telemedicina, teledocencia, teleeducación, realizadas en sedes empresariales e institucionales, y en las que la prestación de servicios se ejecuta a distancia, pero con respecto al cliente y no al empleador. Consiguientemente, el teletrabajo se caracteriza por constituir una forma de organización de la actividad empresarial donde físicamente se distancia al trabajador de la empresa prestando sus servicios desde un lugar distante, con independencia de que se relacione o no directamente con el cliente.

Necesariamente deberán adecuarse las condiciones necesarias incluso de orden ergonómico para que el teletrabajo pueda implementarse y tener éxito en la adaptabilidad laboral. ${ }^{14}$

13 La pandemia sanitaria del COVID-19 provocó que en el mundo entero las empresas, organizaciones, centros de trabajo que se vieron obligadas a cerrar porque sus actividades productivas no se consideraban esenciales, se habilitaron una diversidad de plataformas o aplicaciones a otras que ya se utilizaban de manera cotidiana como medio de comunicación virtual como, Skipe, Facebok, Whatsap, Facetime, correo electrónico; las universidades públicas y privadas al menos en México migraron la terminación del ciclo lectivo 2020 a la modalidad virtual o en línea, en el sistema básico de educación pública se establecieron en canales de televisión estatales, el auge se generalizó para el teletrabajo en aplicaciones y plataformas como zoom, Meat, Youtube, Webwinar, entre otras que al menos han permitido cumplir parcial o totalmente las tareas respectivas.

14 Suárez Barrios, Astrid Sofia, Debates, UNAD, vol. 7, enero-diciembre de 2016, p. 27. 
TABLA 1. Condiciones de éxito del teletrabajo

\begin{tabular}{|c|c|}
\hline Condiciones de éxito & Características \\
\hline Espacio propio & $\begin{array}{l}\text { Lugar que proporcione comodidad, luminosidad y privacidad. } \\
\text { Por otro lado, la familia del teletrabajador debe entender que } \\
\text { éste, aunque se encuentre en el hogar, no está de vacaciones. Por } \\
\text { ende, no debe ser interrumpido en cada momento por temas } \\
\text { domésticos. }\end{array}$ \\
\hline Herramientas TIC & $\begin{array}{l}\text { Contar con elementos tecnológicos como son un notebook, soft- } \\
\text { wares productivos, correo electrónico, telefonía y conexión a In- } \\
\text { ternet }\end{array}$ \\
\hline Orientación a metas & $\begin{array}{l}\text { Lograr los objetivos definidos debe ser el norte para quien se } \\
\text { desempeñe en esta modalidad. Hay que recordar que la evalua- } \\
\text { ción de cada trabajador se hará con base en los resultados que } \\
\text { alcance. }\end{array}$ \\
\hline Sistemático & Ser ordenado y metódico en sus funciones, a lo largo del tiempo. \\
\hline Proactividad & $\begin{array}{l}\text { Tener iniciativa propia, ya sea para las tareas que a diario ejecuta } \\
\text { como para la toma de decisiones o problemas que deba resolver. }\end{array}$ \\
\hline $\begin{array}{l}\text { Capacidad para admi- } \\
\text { nistrar su tiempo }\end{array}$ & $\begin{array}{l}\text { Ser capaz de obtener el mayor provecho de su tiempo, en favor } \\
\text { de la productividad. Ideal es contar con un horario de trabajo } \\
\text { establecido para ello. }\end{array}$ \\
\hline Automotivación & $\begin{array}{l}\text { Tener su motor propio, que le permita superar aquellos momen- } \\
\text { tos difíciles y alcanzar sus metas. }\end{array}$ \\
\hline Contacto Social & $\begin{array}{l}\text { Buscar contactarse con sus compañeros y no perder el vínculo } \\
\text { social con el equipo. }\end{array}$ \\
\hline
\end{tabular}

FUENTE: Suárez Barrios (2015) y adaptado por Toselli (2011).

Asimismo, se ha afirmado que el teletrabajo no se perfila por la distancia como en las actividades anteriores descritas, sino que lo realmente importante es que lo que se realiza es trabajo y, por lo tanto, el trabajo se convierte en un producto en sí mismo, que se puede mover, con el que se negocia y se comercia. ${ }^{15}$

15 Sierra Benítez, Esperanza, El contenido de la relación laboral en el teletrabajo, Sevilla, Consejo Económico y Social de Andalucía, 2011, p. 37. 
Los seres humanos del siglo XXI son de dos mundos, al menos en contexto de sus relaciones sociales y laborales, el mundo en línea (on-line) y el mundo fuera de línea (off-line) que insta e incita a construir nuestros modos y medios, valiéndose de estratagemas, instrumentos ofrecidos por la tecnología informática. ${ }^{16}$

Los tipos de conexión existentes para el teletrabajo pueden ser tres: off-line, one way line y on line, y a través de ellos se transmiten las informaciones relacionadas con el trabajo mediante los medios técnicos, equipos informáticos y de telecomunicaciones que se dispongan. De este modo hacemos referencia al momento cualitativo de la prestación, es decir, al enlace entre el trabajador y la empresa que da lugar a tres posibilidades o tipos de teletrabajo. En segundo lugar, tenemos la conexión one way line, que es aquella en la que el teletrabajador desarrolla su actividad mediante una conexión en sentido estricto entre su videoterminal y el ordenador central, sin un control directo sobre la terminal externa, limitándose a enviar directamente los datos, la vinculación es unidireccional o en sentido único, que bien puede partir del empresario, o alternativamente del trabajador. ${ }^{17}$

En primer lugar está la conexión off-line, en que el teletrabajador desarrolla una actividad informática sin ninguna conexión con el ordenador central situado en la empresa. Este es el motivo por el que algún autor no reconoce como modalidad de teletrabajo aquella que tenga este tipo de conexión, ya que la utilización del correo electrónico no constituye un elemento imprescindible para la realización del trabajo, ni un elemento distintivo con respecto a la realización del trabajo tradicional.

No obstante lo señalado, el trabajador recibe unas instrucciones iniciales y cuenta con un control sucesivo del empresario en relación con los datos enviados que con posterioridad entrega el trabajador, bien por correo, mensajería urgente o personalmente en la empresa. Al no existir conexión directa entre el trabajador y la empresa, el empresario no puede ejercitar continuamente y en tiempo real los poderes de dirección y control. ${ }^{18}$

16 Spitzer, Manfred, Demencia digital, México, Penguin Randow House, 2018, p. 19.

17 Escudero Rodríguez, Ricardo, “Teletrabajo”, cit., p. 769. En Mella Méndez, Lourdes, "Sobre una nueva manera de trabajar: el teletrabajo", cit., p. 45, esta modalidad proporciona al trabajador una cierta libertad que permite al trabajador comunicarse con el empresario cuando lo desee. "La respuesta empresarial parece que, en principio, tiene que efectuarse por una vía diferente (por teléfono, personalmente) a la del video terminal", salvo que se permita la interactividad.

18 Sierra Benítez, Esperanza, op. cit., p. 51. 
En segundo lugar tenemos la conexión one way line, que es aquella en la que el teletrabajador desarrolla su actividad mediante una conexión en sentido estricto entre su videoterminal y el ordenador central, sin un control directo sobre la terminal externa, limitándose a enviar directamente los datos. La vinculación es unidireccional o en sentido único, que bien puede partir del empresario, o alternativamente, del trabajador. ${ }^{19}$

El trabajo en las plataformas suele caracterizarse por remuneraciones inferiores al salario mínimo, flujos impredecibles de ingresos y la ausencia de protecciones laborales que suelen observarse en una relación de trabajo típica.

\section{TECNOLOGÍA Y TECNOTRABAJO}

$\Psi$ 1 espacio electrónico es fácilmente considerado como un evento puramente tecnológico; y en tal sentido como independiente y neutral, ya que es parte de dinámicas mayores que organizan a la sociedad; ya sea en la geografía de su infraestructura o en la estructura del mismo ciberespacio, el espacio electrónico está inscrito — y de alguna manera moldeado- por el poder, la concentración y la oposición, al igual que por la apertura y la descentralización. ${ }^{20}$

De este modo, es por ahora bien conocido que las características particulares del Internet o la red son en parte una función de la temprana cultura hacker de las computadoras, que diseñaron un software que fortaleció la apertura y descentralización de la red y que buscó hacerla universalmente accesible. ${ }^{21}$

El Internet es poder distribuido por la ausencia de jerarquías, sus atributos personales han engendrado además de un poder que cuenta con deslocalización, descentralización, apertura, posibilidad de expandirse en jerarquías, sin centros ni condiciones para el control autoritario o monopólico. Donde además las redes electrónicas están haciendo otras formas de poder, sobre todo enmarcados en temas financieros, como las tres propiedades de las redes electrónicas: velocidad, simultaneidad e interconectividad.

En la incorporación del espacio electrónico y digital para el teletrabajo, se pueden integrar factores como:

\footnotetext{
19 Ibidem, p. 52.

20 Lessa Kerstenetzy, Celia, El Estado de bienestar social en la edad de la razón, México, Fondo de Cultura Económica, 2017, p. 63.

21 Lozano Chairez, Miguel, Sombreros blancos, tres hackers programan un mundo mejor, México, Universidad Autónoma de Baja California, 2017, p. 39.
} 
1. No hay empresa completamente virtualizada, ni industria totalmente digitalizada, ni trabajo formal hoy que pueda que contener la dualidad de ser virtual y digital, los sectores económicos líderes que están altamente digitalizados requieren de sitios estratégicos con vastas concentraciones de infraestructura, el requisito de fuentes laborales, talento y edificios; esto es totalmente válido para las empresas de finanzas, pero también para las industrias multimediáticas, que usan procesos de producción digital y productos digitalizados.

2. Las crecientes desigualdades en la distribución de la infraestructura para el espacio electrónico, ya sean redes de computadoras privadas o la red global, en las condiciones para el acceso electrónico cuyas condiciones para el acceso a segmentos y características de elevado poder, están todas contribuyendo a nuevas geografías de centralidad tanto territorialmente como en el espacio electrónico.

3. La comercialización de redes públicas y las concentraciones jerárquicas de poder en las redes privadas están produciendo además cibersegmentaciones como manifestaciones de las dinámicas de desigualdad y poder.

Se ha superado la barrera del espacio, no se trata de lo remoto o de la distancia, sino que habrá que referirse a que un nuevo universo laboral es el que implica a todo trabajo realizado desde un lugar no fijo con total independencia del concepto de ajenidad y con la exclusividad puesta en el uso de lo digital.

En la actualidad se pueden identificar tres vertientes de la comprensión del concepto de inclusión digital: acceso, alfabetización digital y apropiación de tecnologías. La primera se basa en la distribución de bienes y servicios que garanticen el acceso a la infraestructura y a las tecnologías de información y comunicación.

La segunda vertiente enfatiza las habilidades básicas en las mismas herramientas que permitan al individuo saber hacer uso de ellas y, en este caso, el acceso a los medios físicos y la alfabetización escolar representan los requisitos necesarios a desarrollar. ${ }^{22}$

El trabajo digital es la expectativa de lo que es el trabajo en que la pandemia del COVID-19 establece que se desarrollará en el presente y futuro;

22 Disponible en: http:// mwm.conectas.org/revista-sur/conectas-e-fundacao-carloschagasdivulgamsele cionados-para-o-programa-de--incentivo-a-producao-academica-em-direitoshumanos? pg. $=2$, consultado el 3 de junio de 2020. 
es labor basada en el talento humano, para que sea cambiante, adaptado a la conectividad, competitivo, digital, inteligente, flexible y medido en resultados. De igual manera, las nuevas tecnologías han afectado el trabajo generando mayor desempleo y exclusión social.

El único hombre educado para el trabajo es aquel que ha aprendido a aprender, el hombre que ha sabido adaptarse a los cambios, que ha llegado a darse cuenta que ningún conocimiento es seguro y comprende que solamente el proceso de saber buscar este conocimiento, le dará seguridad. ${ }^{23}$

Un espacio de trabajo digital es un marco de trabajo de tecnología integrado, diseñado para entregar y administrar la entrega de aplicaciones, datos y escritorios. Permite a los empleados acceder a sus aplicaciones y datos en cualquier dispositivo, desde cualquier lugar. ${ }^{24}$

Las tecnologías de la información y la comunicación tienen las principales características que lo determinan así:

- Son innovadoras y creativas, pues facilitan el acceso a nuevas o novedosas formas de comunicación.

- Tienen más influencia y benefician en mayor proporción al área educativa, haciéndola más accesible y dinámica.

- Afectan a numerosos ámbitos de las ciencias humanas como la sociología, la teoría de las organizaciones, la medicina, el derecho y la biología entre otras.

- Descongestionan los costos económicos de una organización a largo plazo.

- Constituyen medios de comunicación y adquisición de información variada, inclusive científica, a las cuales las personas pueden acceder por sus propios medios; es decir, potencian la educación a distancia.

- Facilitan la creación de nuevos puestos de trabajo, además de reconvertir los ya existentes.

Las diferencias en el acceso y usos de las tecnologías como herramientas para la apropiación del conocimiento generan nuevas problemáticas sociales, que requieren de una seria reflexión acerca de las oportunidades, limitaciones y dificultades que brindan las tecnologías, si bien es cierto que la digitalización

23 Garza Rosa, Leventhal Susana, Aprender cómo aprender, México, Trillas, 1998, p. 11.

24 Disponible en: https:// www.citrix.com/es-mx/glossary/what-is-digitalworkspace.html\#: $\sim:$ text $=U n \% 20$ espacio\%20de\%20trabajo\%20digital, consultado el 12 de junio de 2020. 
proporciona ilimitadas oportunidades, no se puede soslayar que las emociones que trasmite no son las mismas; al final, el ser humano es un ser de afectos y de relaciones sociales.

La consecuencia para obtener las ventajas o desventajas que implica la inserción al teletrabajo, se diseña con una matriz estructurada. ${ }^{25}$

TABLA 2. Posibles ventajas e inconvenientes del teletrabajo

\begin{tabular}{|c|c|c|}
\hline \multicolumn{3}{|c|}{ Posibles ventajas e inconvenientes del teletrabajo } \\
\hline & Ventajas & Inconvenientes \\
\hline \multirow{6}{*}{ Para el individuo } & Mejor rendimiento y productividad & $\begin{array}{l}\text { Menos oportunidad para las rela- } \\
\text { ciones personales }\end{array}$ \\
\hline & $\begin{array}{l}\text { Menos tiempos de desplazamiento } \\
\text { al trabajo }\end{array}$ & $\begin{array}{l}\text { Menos estrés relacionado con el } \\
\text { hogar }\end{array}$ \\
\hline & Mayor autonomía & Más aislamiento profesional \\
\hline & Menor estrés laboral & Menos seguridad con empleo \\
\hline & Más tiempo con la familia & \\
\hline & $\begin{array}{l}\text { Única posibilidad de ingreso en el } \\
\text { mercado laboral (persona con hijos } \\
\text { pequeños, discapacidades) }\end{array}$ & \\
\hline \multirow{5}{*}{$\begin{array}{l}\text { Para la organiza- } \\
\text { ción }\end{array}$} & Mayor productividad & $\begin{array}{l}\text { Desafío a la posibilidad de control } \\
\text { y motivación de los teletrabajado- } \\
\text { res }\end{array}$ \\
\hline & $\begin{array}{l}\text { Oferta de trabajo más amplia y va- } \\
\text { riada }\end{array}$ & Empleados menos comprometidos \\
\hline & Ahorro de espacio de oficina & Pérdida de trabajo en el equipo \\
\hline & Menos ausentismo & \\
\hline & Imagen de empresa flexible & \\
\hline
\end{tabular}

25 Suárez Barrios, Astrid Sofia, op. cit., p. 28. 


\begin{tabular}{|l|l|l|}
\hline \multirow{2}{*}{$\begin{array}{l}\text { Para la socie- } \\
\text { dad }\end{array}$} & Menos desplazamiento & $\begin{array}{l}\text { Individuos más aislados de las } \\
\text { instituciones sociales }\end{array}$ \\
\cline { 2 - 3 } & Menos contaminación & Sociedad autista \\
\cline { 2 - 3 } & $\begin{array}{l}\text { Menos congestión de tráfico y ac- } \\
\text { cidentes }\end{array}$ & \\
\cline { 2 - 3 } & Menos discriminan laboral & \\
\hline
\end{tabular}

FUENTE: Baruch (2001) y ajustada por Suárez-Barros (2015).

Las tecnologías digitales se han convertido en una fuerza poderosa para el desarrollo social y económico. Las características cambiantes de nuestra sociedad, que en gran parte se deben al acceso generalizado a las tecnologías y a la información, se reflejan en cuestiones de cómo, cuándo y dónde enseñar y aprender. ${ }^{26}$

Las tecnologías de la información y la comunicación, tecnologías del conocimiento, permiten innovar, recrear realidades, acercar mundos y, sobre todo, a las personas. Esta amalgama de saberes y experiencias sin ninguna duda requiere de nuevos aprendizajes que conjuguen no solo aspectos instrumentales de conocimiento de las herramientas, sino de maneras diferentes de observar, de conocer, de relacionarse e interactúa en un mundo en que las TIC mimetizan nuestra vida cotidiana. ${ }^{27}$

Cada revolución tecnológica conlleva una reorganización del sistema socioeconómico en que la revolución de la información ha sido un factor de suma importancia que ha alterado la manera en cómo las personas adquieren y hacen transferencia del conocimiento. La comunicación y el lenguaje, el uso mismo de las TIC ha afectado a la vida privada, laboral y profesional de las personas en su entorno cotidiano.

Las tecnologías son poderosas herramientas que admiten nuevas formas de comunicación, de aprendizaje de socialización, incluso de divertimento, es decir es un nuevo medio de cultura. ${ }^{28}$

26 Castell, M., La era de la información, economía, sociedad, cultura, Madrid, Alianza,1999, p. 145.

27 Garzón, Clemente y Gómez, Natalia, Alfabetización laboral, México, Plaza y Valdés, 2018, p. 19.

28 Se podrá considerar una nueva generación de la pandemia como del confinamiento o del aprendizaje digital, por ello el complemento será la lectura digital que se realiza a través de la pantalla de un dispositivo tecnológico, como una computadora, teléfono móvil o lectores digitales. 
Por ello, el avance de la tecnología ha llegado a alcanzar un escenario de dimensión preocupante e incluso deshumanizadora con dos ópticas; la primera, de estar conectados a la red mediante los dispositivos celulares que permiten estar vinculados en todo momento y en cualquier lugar; lo cual supone establecer una continuidad sobre actuaciones vinculadas en la actividad laboral durante incluso la jornada de trabajo y, la segunda, esa delgada línea que separa la conexión laboral digital, que separa el tiempo de trabajo y el tiempo de descanso.

En plena era de las comunicaciones, en la que el Internet y las redes sociales, en principio, son lugar común de la inmensa mayoría de los ciudadanos de los Estados nacionales, se pudiera pensar que, en todo Estado constitucional, los destinatarios de la norma y la autoridad encargada de ejercerla deberían estar muy familiarizados con su contenido y alcance. Esto, técnicamente facilitaría la consolidación del llamado Estado de derecho, de esta forma, los derechos humanos y las instituciones encargadas de aplicarlos constituirían un lugar común y cotidiano en las democracias constitucionales, lo paradójico resulta cuando contrastamos la realidad sociopolítica con los logros en materia de justicia social y de respeto a la esfera jurídica del gobernado, esto es, de la eficacia de la Constitución.

La vida cotidiana se ha ido simplificando significativamente desde la aplicación de la tecnología y lo digital, lo analógico se ha quedado de lado de manera que lo más pequeño, lo más cómodo e inclusive lo más práctico es netamente digital. Lo digital con todas sus propias implicaciones es ya un término que define al cualquier maquinaria que sea computarizada, no importa su naturaleza, el hombre se ha dedicado a estructurar un sistema digital para vivir en él, lo básico es digital, lo complejo también lo es.

La tecnología existe desde antes incluso que la escritura; el término se refiere tanto a artefactos materiales como a procesos que resuelven problemas; la sociología de la tecnología pretende comprender el uso y cambio tecnológico desde una perspectiva amplia que recupere visiones de diferentes disciplinas.

Para contrarrestar perspectivas simplistas del desarrollo tecnológico, por ejemplo, el determinismo tecnológico, que asume que los inventos e innovaciones son producto de mentes geniales cuyas ideas crean una discontinuidad respecto al pasado, desde estas posturas, los nuevos inventos son innovadores porque no tienen relación con lo que se ha hecho anteriormente. ${ }^{29}$

29 Basalla, G., La evolución de la tecnología, Barcelona, RBA, 1999, p. 74. 
Por otro lado, la sociología de la tecnología parte de la premisa de que la tecnología es una construcción social y, al ser producto del ser humano, debe comprenderse en relación con su contexto e historia. La evolución de la tecnología afirma que es posible comparar la tecnología de la evolución de Darwin con los procesos que han ocurrido en el avance de la tecnología.

Es decir, todos los artefactos actuales provienen de un anterior y la adaptación de éstos puede explicarse por muchos factores. Por decirlo como metáfora; las computadoras actuales remiten a antiguas máquinas de escribir del siglo XIX: ninguna sociedad está aislada o es autosuficiente de forma que nunca haya tomado prestados al menos algunos aspectos de su tecnología de una fuente exterior. ${ }^{30}$

Los motivos del cambio tecnológico son sociales, económicos, ambientales, sicológicos, culturales y migratorios, pero siempre existe un precedente: para que se dé un cambio evolutivo, la novedad ha de hallar una forma de afirmarse en medio de la continuidad.

La tecnología se construye socialmente en su práctica y avance como parte de la vida social; así, la sociedad no es considerada algo aparte de la naturaleza, sino como sistemas que interaccionan y pueden modificarse mutuamente. La tarea de la antropología social del mundo moderno consiste en describir de la misma manera el modo en que se organizan las ramas de nuestro gobierno, inclusive la de la naturaleza, las ciencias exactas, explicando de qué manera y por qué esas ramas se separan, así como los múltiples arreglos que la reúnen para que se dé un cambio evolutivo, la novedad ha de hallar una forma de afirmarse en medio de la continuidad. ${ }^{31}$

La tecnología es un objeto tangible que puede medirse y razonarse, forma parte del sistema cultural, es un signo con significante y significado del cual no es posible extirpar toda la carga subjetiva o simbólica; esto quiere decir que se debe aprender a ver la tecnología como un discurso, como un sistema de signos, sujeto a fuerzas semióticas y que responde a demandas sociales y sicológicas. ${ }^{32}$

Las nuevas tendencias para el desarrollo de actividades surgen principalmente en los campos vinculados a la tecnología, la innovación, la robotización, las plataformas digitales, así como de la informática, donde se generan

\footnotetext{
30 Ibidem, p. 88.

31 Idem.

32 Alexander, J. C., "Sociología cultura, lo sagrado y lo profano en el discurso tecnológico", Revista Mexicana de Sociología, 1991, p. 296.
} 
formas de trabajo no presencial, sin ubicación local, que constituyen una prolongación o diversificación de ciertas funciones empresariales hacia los trabajadores que, incluso, llegan a trascender países.

Las tecnologías de la información y la comunicación también son el conjunto de tecnologías desarrolladas para almacenar, gestionar, recuperar, recibir y enviar información de un sitio a otro, o procesarla para poder calcular resultados y elaborar informes, que comprenden las tradicionales tecnologías de la comunicación, que se integran por la televisión, la radio y la telefonía, y las tecnologías de la información, caracterizadas por la digitalización de los registros de contenidos (informática, de las comunicaciones, telemática y de interfaces).

Lo imperante rompe con el sistema clásico de relaciones laborales y da lugar a cambios trascendentales, estos cambios han dado paso a una descentralización del trabajo generando ahorro de tiempo y recursos, con implícitas transformaciones técnicas, económicas y sociales, dando lugar a la incertidumbre laboral de un conglomerado considerado vulnerable dentro de la propia clase trabajadora.

Lo que caracteriza a la sociedad actual en su desarrollo en lo referente a la información, es la modificación de las características y posibilidad de acceso a la misma en nuevos formatos y facilidad de expansión a través de tecnologías y procesos informáticos de telecomunicaciones y digitalización. Estas posibilidades son la esencia configuradora de la idiosincrasia del momento social, ya que las actividades de reproducción primaria y de transacción, de cualquier tipo, se realizan a partir de información, principalmente digital y procedente de Internet. ${ }^{33}$

Sabedor debe estar el imaginario colectivo de que el uso y la aplicación de nuevas tecnologías tiene diversos efectos transversales en todos los ámbitos de nuestra sociedad, que del mismo modo estas facilidades en el acceso se han vuelto los principales obstáculos para establecer la adecuada conciliación entre los límites efectivos en la vida laboral y personal y familiar de la gente que trabaja.

Los procesos de flexibilización permanentes, el desarrollo de las nuevas tecnologías, el riesgo deshumanizante de las relaciones de trabajo, la diversidad en la oferta de la oportunidad de búsqueda de empleo, ${ }^{34}$ así como di-

33 Hernández-Serrano, M. J., Estrategia de búsqueda de información para la generación de conocimiento, Red de Universidad de Salamanca, 2009, pp. 24 y 25.

34 Se puede entender que el empleo es al menos la búsqueda focalizada en el entorno llamado mercado de acceso a determinada actividad, es la posibilidad de acceder al trabajo for- 
versas causas multifactoriales, la interrogante y la expectativa por las secuelas de la pandemia sanitaria del COVID-19, ¿hasta dónde llegará esta realidad imperante? Derivado del nuevo escenario para las relaciones laborales, donde a la sociedad global se le ofrece una nueva regularidad, la pandemia de la afectación de la salud laboral coexistirá con nuevas formas y procedimientos, donde los factores deseados es que equilibren este escenario, donde la regla sea extender, contraer, recortar, pero nunca romperse, ante el desarrollo de la misma sociedad productiva.

Lo que implica reflexionar sobre las ventajas de la digitalización en relación con el derecho de los trabajadores del descanso después de su jornada laboral, es decir, limitar el espectro legal o convencional, para evitar situaciones de exceso de sobrecarga laboral, durante los tiempos de vida privada, donde la desconexión laboral debe imperar, salvo contingencias o imprevistos de manera casual o fortuita. Siempre y cuando no se trate de trabajadores considerados en la modalidad de teletrabajo o con diversas acepciones propias de la naturaleza del trabajo que se presta. Al menos en México esto no está previsto frente al desbordado y avasallante avance de la tecnología y la digitalización social, por ello es imperante considerar el derecho relacionado con la conexión laboral y más aún por las vías digitales.

En el ámbito de los convenios de la OIT, tampoco se tienen previstas esas disposiciones respecto de la regulación del derecho para la conexión y/o desconexión digital, que se pueden dar a través del teléfono, el ordenador o cualquier otro dispositivo electrónico y digital fuera de la jornada ordinaria de trabajo. ${ }^{35}$

Los principales cambios en las organizaciones derivados de la inseguridad económica, así como de la pobreza centrada en el empleo, se limita a tres

mal que garantice derechos, exija obligaciones y configure responsabilidades de la más amplia protección social.

35 En el "Informe sobre el futuro del trabajo", Rebeca Grynspan, secretaria general Iberoamericana en febrero de 2019 en Lima, Perú, presentó el informe de la Comisión Mundial sobre el futuro del trabajo de la OIT, que integran 27 países, planteó que "El futuro del trabajo no depende de la tecnología, depende de nosotros", haciendo alusión en un programa para generar empleo basado en la inversión de las capacidades de los individuos, en las instituciones laborales, en el trabajo decente y sostenible, donde a pesar del catastrofismo de que millones de oficios puedan ser sustituidos, por lo tanto se debe invertir en nuevos derechos, nuevos mercados, nuevas ideas y nuevas personas, con una visión próspera e inclusiva; el futuro del trabajo es una oportunidad no un coste, entendiendo que detrás de toda tecnología hay una mano humana, donde el tripartismo y el diálogo social siguen siendo la forma más eficaz de enfrentar estos cambios. 
procesos: 1) la creciente desigualdad en las capacidades de producción de ganancias de diferentes sectores económicos y en las capacidades de obtención de ingresos de los distintos tipos de trabajadores; 2) las tendencias a una polarización incorporada en la organización de las industrias de servicio y en la precarización de la relación del empleo, y 3) la producción de una marginalidad urbana, particularmente como resultado de nuevos procesos estructurales de crecimiento económico.

Desde la perspectiva de la diversidad del empleo, donde la exclusión tiene predominio en el área urbana de las ciudadades: 1) el predominio y la trasformación de las finanzas, en especial a través de la sectorizacion, la globalización y el pleno desarrollo de nuevas telecomunicaciones y tecnología de sistemas informaticos; 2) la creciente intensidad de servicios en la organización de la economía, que generalmente ha elevado la demanda de servicios por parte de las empresas y hogares. Por ello la calidad del empleo refiere tres dimensiones: el aspecto económico, el de la calidad y el que abarca los aspectos institucionales.

TABLA 3. Factores determinantes de la calidad en el empleo

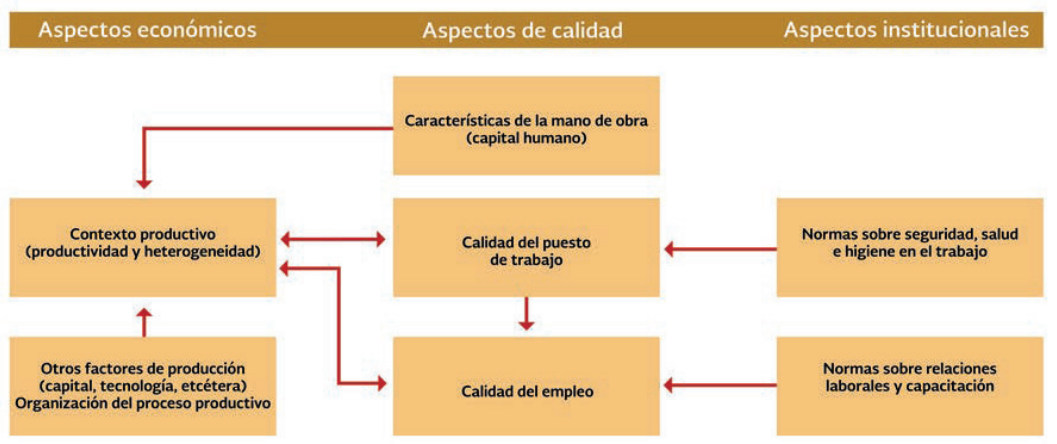

FUENTE: Elaboración propia con base en el estudio sobre inclusión laboral, avances y retos, STPS 2015.

Dentro de las cadenas de valor de la integración laboral y la cibersegmentación, se destacan tres nuevas condiciones:

1. La creciente digitalización y globalización de los sectores económicos líderes ha contribuido a la hipercontración de recursos, infraestructura 
y funciones centrales, donde las ciudades son los sitios estratégicos en la red económica global.

2. La creciente importancia económica del espacio electrónico ha fomentado alianzas globales, concentraciones masivas de capital y de poder corporativo.

3. Nuevas formas de segmentación en el espacio público y privado.

\section{DISRUPCIÓN POR TELETRABAJO}

Si in lugar a dudas, la era de la información ha dado paso a la era del conocimiento y a su vez los desarrollos tecnológicos han producido cambios no sólo en el ámbito del trabajo sino también sobre las pautas que influyen en la forma de vivir de la sociedad productiva, afectada de manera integral por la calamidad sanitaria del COVID-19, de igual manera por la crisis brutal económica de la recesión mundial, el teletrabajo permite conservar los puestos de trabajo y hasta incrementarlos sin optar por la variante histórica del recorte de la mano de obra por despidos.

Lo que ha sucedido en nuestro país es que las reformas han recurrido al esquema de reducir el empleo, el salario y la protección social de sus trabajadores. Mediante la flexibilización de la legislación se ha logrado reducir el costo laboral, lo que no ha cambiado es el entorno del trabajo; aun reduciendo ese costo, los problemas en el mercado del trabajo se han agravado. Si la economía mexicana se encuentra estable en el discurso, pero en grave recesión, por la pérdida de miles de empleos en cifras y resultados, ¿por qué existe entre la población una sensación de malestar económico generalizado de no contar con ingresos suficientes o básicos? o, en el peor de los casos, ¿hacia dónde se está dirigiendo el bienestar que le corresponde a la población por el retroceso económico por mínimo que parezca o resulte?, lo anterior porque la pandemia del coronavirus provocó el cierre total y parcial de actividades productivas, la suspensión de empresas, establecimientos y la parálisis del trabajo .

La revolución tecnológica de la mano de la pandemia de salud que provocó el COVID-19, ha echado del mercado de trabajo a miles de humanos; que los gobiernos que no sepan encarar estos desafíos de la estructura social, seguramente originará revueltas sociales y políticas que ninguna ideología existente sabrá cómo manejar, todos los debates sobre tecnología e ideología pueden parecer abstractos y lejanos, pero la perspectiva del desempleo masivo o desempleo personal por la tecnología y la pandemia no dejará indiferente a nadie. 
Los temores de que la automatización podría generar un desempleo masivo se remontan al siglo XIX, hoy por la pandemia del COVID-19 se visualiza una rápida materialización para ello, hay razones para pensar diferente y que la opción de un aprendizaje automático de lo digital conllevará adecuaciones y/o adaptaciones.

La intervención de la fuerza de trabajo es indispensable, puesto que no sólo se trata de un activo más de la empresa que se debe plasmar en una filosofía que valore los recursos humanos. Se debe tener en cuenta también que la motivación de las personas no solamente reside en el dinero, sino también en potenciar las capacidades intelectuales, ambientes de trabajo agradables, promociones dentro de la empresa. De la población económicamente activa entre la población ocupada y desocupada con datos hasta el año 2018 — sin duda el impacto del desempleo y la inserción al teletrabajo se reflejarán conforme a las proyecciones demográficas - la tabla 4 dará un matiz diferente de país durante la pandemia y las secuelas que arrastrará el mercado del empleo y del trabajo del año 2020 en adelante.

TABLA 4. Población según su condición de actividad (personas)

\begin{tabular}{|c|c|c|c|c|c|}
\hline \multirow[b]{2}{*}{ Indicador } & \multicolumn{5}{|c|}{ Primer trimestre } \\
\hline & 2017 & 2018 & Diferencias & $\begin{array}{c}\text { Estructura } \\
\% 2017\end{array}$ & $\begin{array}{c}\text { Estructura } \\
\% 2018\end{array}$ \\
\hline Población total & $123^{\prime} 057,147$ & $124 ’ 286,623$ & 1'229,476 & & \\
\hline Población de 15 años y más & $90^{\prime} 644,546$ & $92 ' 604,805$ & 1’960,259 & 100.0 & 100.0 \\
\hline $\begin{array}{l}\text { Población Económicamente } \\
\text { Activa (PEA) }\end{array}$ & $53^{\prime} 681,720$ & $54,590,773$ & 909,053 & 59.2 & 59.0 \\
\hline Ocupada & $51 ' 859,895$ & $52 ’ 876,916$ & 1’017,021 & 96.6 & 96.9 \\
\hline Desocupada & 1’821,825 & $1 ’ 713,857$ & (-) 107,968 & 3.4 & 3.1 \\
\hline $\begin{array}{l}\text { Población No Económica- } \\
\text { mente Activa (PNEA) }\end{array}$ & $36 ’ 962,826$ & $38^{\prime} 014,032$ & 1’051,206 & 40.8 & 41.0 \\
\hline Disponible & $5 ’ 738,293$ & $5 ’ 784,558$ & 46,265 & 15.5 & 15.2 \\
\hline No disponible & $31 ' 224,533$ & $32 ’ 229,474$ & 1’004,941 & 84.5 & 84.8 \\
\hline
\end{tabular}

FUENTE: INEGI, México. ${ }^{36}$ Los datos absolutos de las encuestas en hogares se ajustan siempre a proyecciones demográficas que son generadas por el Consejo Nacional de Población.

36 Los datos están ajustados a las proyecciones demográficas del Consejo Nacional de Población, 2010-2050, publicadas el 16 de abril de 2018. 
La utilización de tecnología moderna necesaria de implantar en América Latina, conlleva una cultura de productividad disruptiva en materia de prevención, adecuación en los esquemas de salud e higiene para el trabajo propios de los empresarios y también entre los trabajadores. Además de invertirse en la formación integral de los recursos humanos, debe mejorar la organización del teletrabajo dentro de las empresas. ${ }^{37}$

Todo ello porque se pasa más tiempo en el centro de trabajo que en los propios hogares, en consecuencia, el adelantamiento económico debe ir acompañado también del progreso social para enfrentar los desafíos de la productividad y buscar que los derechos de los trabajadores reinsertados en la modalidad del teletrabajo, no se vean mermados con los cambios en la economía de afectación y estrategias de crecimiento.

Las actividades que pueden difícilmente evidenciar al teletrabajo como la forma más recurrente del cambio en las relaciones laborales se pueden clasificar con base en dos criterios: 1) por un lado, atendiendo a la naturaleza de la actividad realizada, y 2) por otro, atendiendo al grado de desarrollo de la actividad formal, lo que implica que las actividades informales y las empresas informales hacen imposible la identificación de dicho sector aun cuando se establecen obligaciones legales para cumplir. ${ }^{38}$

Entre las desventajas que se le atribuyen al teletrabajo encontramos las siguientes:

a) Precarización del empleo.

b) No realización en ocasiones del trabajo en equipo.

c) Aislamiento del teletrabajador.

d) Falta de supervisión directa y presencial de la empresa.

e) En ocasiones bajo nivel de compromiso empresarial.

f) Violación de derechos laborales.

g) Requerimiento de autodisciplina.

h) Imposibilidad de ascensos en la organización.

37 Tokman, Víctor, "Políticas de empleo en la nueva era económica", Economía, Lima, vol. XXIII, núm. 46, 2000, p. 25.

38 En la Ley Federal del Trabajo de México, el artículo 318 anota: "Las condiciones del trabajo se harán constar por escrito. Cada una de las partes conservará un ejemplar y el otro será entregado a la inspección del trabajo. El escrito contendrá: Nombre, nacionalidad, edad, sexo, estado civil, y domicilio del trabajado y del patrón; I. Local donde se ejecutará el trabajo... III. Naturaleza, calidad y cantidad del trabajo; IV. Monto del salario fecha y lugar de pago, y V. Las demás estipulaciones que convengan las partes". 
i) Reticencia a la capacitación por parte del trabajador.

j) Peligros derivados de la confidencialidad de la información proporcionada.

k) Problemas de salud por falta de mobiliario ergonómico, entre otras.

1) Deficiente conectividad de Internet.

\section{MARCO JURÍdiCO EN MÉXICO}

$\mathrm{E}$ n México, derivado de la reforma laboral de 2012, se ha contemplado que en la actualidad no se trata de una nueva figura de trabajadores sino simplemente de una forma de organización del trabajo y, por lo tanto, la normatividad jurídica aplicable al trabajo a domicilio es, por consiguiente, la relativa al teletrabajo. ${ }^{39}$

El artículo 311 es el que regula el trabajo a domicilio, el cual es el que habitualmente se presta en el domicilio de cualquiera o en un local libremente elegido, donde sin vigilancia, ni dirección ni subordinación directa o indirecta de quien proporciona el trabajo o alguna actividad a desempeñar. En la inocua y limitada reforma laboral llevada a cabo, se agregó a lo que se considera como trabajo a domicilio el que se realiza a distancia utilizando tecnologías de la información y la comunicación, de donde se infiere que se trata de teletrabajo, aun cuando carece de una definición propia. Lo cual deja más interrogantes que certeza jurídica laboral para este tipo de trabajo ante la pandemia sanitaria del COVID-19, que se caracteriza como atípico, informal, inseguro e incómodo.

Las diversas modalidades de contratación hacen que el teletrabajo aparezca más bien como una versión moderna del antiguo esquema de trabajo a

39 En México el trabajo legislativo de armonización y actualización de la legislación laboral, incurre en la procrastinación (posponer lo importante) para legislar en materia laboral, donde por ejemplo no se contempla actualmente el concepto de teletrabajo, teletrabajador, así como diversas características que conlleva esta modalidad de trabajo a distancia y basado en tecnologías de información, lo que existe de manera parcial es un dictamen aprobado por la comisión del trabajo del Cámara de Senadores del 11 de abril de 2019, que no ha sido aprobada por el Pleno de la misma cámara legislativa, en el referido dictamen establece la inserción del capítulo XII bis en la Ley Federal del Trabajo vigente, que contempla la adición de los artículos $330 \mathrm{~A}$ al $330 \mathrm{E}$, donde se destaca la conceptualización del teletrabajo y otros aspectos descriptivos irrelevantes jurídicamente; sin embargo, no se consideran las circunstancias que la pandemia del COVID-19 refiere en el tema de salud y seguridad para el trabajo por las condiciones sanitarias. 
domicilio, y coincide con éste en que la labor se realiza fuera del espacio físico de la empresa, generalmente en un espacio remoto como Internet y en general con apoyo de sistemas de cómputo/informática, para estar "conectados" y relacionados con la empresa. En estos casos, la manera como se presta el trabajo impide la aplicación de la normatividad laboral en atención a que se aleja de los conceptos que la misma plantea. ${ }^{40}$

Si la adecuación de la normativa laboral precisa objetividad y puntualidades, resulta sin duda indispensable precisar que se garantiza el derecho al trabajo, con promoción y respeto de los propios derechos humanos laborales y de seguridad social, por ello lo que ya de suyo garantiza se deben describir con precisión meridiana en qué consiste el teletrabajo que implique la telerelación laboral que implica telederechos, teleobligaciones y teleresponsabilidades propias e inherentes con telecontrato; que incluya las condiciones de trabajo como la telejornada, teledescansos, telesalario, telecapacitación, teleaguinaldo, telederechos y la forma que garantice sin duda la resolución de sus propios teleconflictos que derivan en teledemandas, telejuicios, telerecursos y teleamparos. Que de igual manera crea telejuntas de conciliación y arbitraje, telecentros de conciliación y registro laboral, teletribunales, ${ }^{41}$ que emitirán teleresoluciones, teleacuerdos, teleconvenios, telelaudos, telesentencias. ${ }^{42}$

Con lo cual todo se transforma en un telegobierno que no ve más allá de su propio interés de gobernar sin ser un Estado pleno, que garantice la sobrevivencia de millones de trabajadores precarizados, donde la telepandemia sanitaria le obliga adecuar todo el bloque normativo respecto de las relaciones laborales, que hoy en la pandemia del COVID-19 resulta de extrema urgencia

40 Reynoso Castillo, Carlos, Los contratos de trabajo, México, UAM Azcapotzalco, 2011, p. 69.

41 En México se encuentra aprobada la reforma constitucional de 2017 que desaparece las juntas de conciliación y arbitraje para la resolución de los conflictos laborales, que hoy dependen del Poder Ejecutivo, se crean para sustituirlos centros de conciliación como etapa prejudicial, de igual manera se crean los tribunales laborales que dependerán del Poder Judicial sea en la competencia federal o en la jurisdicción de las 32 entidades federativas, de igual manera está creado un centro federal para registro de sindicatos y contratos colectivos, la reforma citada se encuentra en implementación programada al mes de octubre del año en curso [2020], pero por la pandemia del COVID-19 aún se encuentran cerrados los existentes para el desahogo de los juicios ya vigentes y los nuevos acumulados que se atenderán por miles derivado del cierre de empresas.

42 En diversas juntas de conciliación y arbitraje de las 32 entidades federativas, debido a la exponencial saturación por la suspensión, modificación y terminación de trabajo, se establecen por videollamadas, por correo electrónico o aplicación digital, conciliaciones laborales para resolver juicios en trámite, hacer convenios de condiciones de trabajo, se agendan, se resuelven y en fecha posterior se ratificarán por escrito en las propias juntas de conciliación. 
social generalmente para todos aquellos que no cuentan con protección jurídica laboral ni con los beneficios que de ella derivan, por estar enfermos y desempleados como consecuencia del cierre total y parcial de centros de trabajo, no contando además con la seguridad social básica e indispensable, para lo cual por la propia reforma que se necesita, se incluya que habrá que reconocer que la telesubcontratación misma, no se hará con trabajadores bajo el esquema de teleoutsourcing.

Lo grave es que ni siquiera hoy es posible tener focalizados los centros de trabajo para una inspección laboral, función propia de una gestión gubernamental que lejos de tener presencia y eficacia en los centros de trabajo de todo el país, ha sido rebasada, por lo que se ha vuelto inoperante y limitada. ${ }^{43}$ En la sociedad actual, en la que el crecimiento tecnológico está dirigido a la eficiencia económica y al ahorro de tiempo, distancias, recursos y esfuerzo, han surgido nuevas formas de trabajo determinadas por el desarrollo de las tecnologías de la información y la comunicación.

Los principales retos del derecho laboral globalizado sea el de adaptar y actualizar no sólo la semántica jurídica, sino también los principios rectores integradores como la contratación colectiva internacional, la migración laboral controlada, los cambios en la administración del trabajo, las nuevas formas de contratación laboral y la flexibilización de los derechos laborales entre otros. ${ }^{44}$

\section{CONCLUSIONES}

$\mathrm{P}$ rimera: la incursión de México —o la inclusión en la ley laboral— en el teletrabajo como trabajo formal es todavía es incipiente, limitada e incompleta aun cuando exista sólo considerar al teletrabajo como trabajo a domicilio; el estudio, cualquier análisis se dificulta toda vez que no existen

43 Incluso se reconoce que existe un vacío de información y armonización, el departamento de condiciones de trabajo e igualdad de la OIT, se encuentra desde 2015 en colaboración con Eurofound realizando estudio en 10 países miembros de la Unión Europea, en la que destaca la utilización de nuevas tecnologías para el trabajo efectuado fuera de los locales del empleador y sus repercusiones en el tiempo de trabajo, la conciliación entre la vida laboral, la vida privada, el rendimiento en el trabajo y la salud, así como el bienestar de los trabajadores, donde debido a las variaciones que impliquen resultados verificables provocadas por el tamaño del muestreo, la ubicación, el sector laboral, así como el nivel de análisis con enfoque teórico y metodología que sea fiable.

44 Kurczyn Villalobos, Patricia et al., Derecho laboral globalizado, México, UNAM, Instituto de Investigaciones Jurídicas, 2007, p. 5. 
estadísticas, datos, inspección y hasta una sobrada irresponsabilidad gubernamental y social ya que sin duda algunas empresas, instituciones y patrones han incursionado en dicho rubro de manera desatinada, o apresurada por el entorno mismo por la pandemia sanitaria del COVID-19.

Segunda: tradicionalmente se desarrolló la idea de que las relaciones laborales en México en general se desempeñan de forma personal, el hecho de que los trabajadores tengan la oportunidad de trabajar lejos de los centros de trabajo, utilizando la red de redes, origina un vacío legislativo que norme esa forma novedosa de trabajo digital, el problema que subsiste en concreto es que la ausencia de normas jurídicas aplicables lleva a la arbitrariedad y deja a estos nuevos trabajadores del teletrabajo a tener perjuicios en su economía ya que deja al patrón un margen de discrecionalidad que es utilizado a su beneficio y perjudica al teletrabajador y en consecuencia casi a la sociedad misma.

\section{FUENTES}

\section{Bibliográficas}

ALEXANDER, J. C., "Sociología cultura, lo sagrado y lo profano en el discurso tecnológico", Revista Mexicana de Sociología, 1991.

BASALLA, G., La evolución de la tecnología, Barcelona, RBA, 1999.

CASTELl, M., La era de la información, economía, sociedad, cultura, Madrid, Alianza, 1999.

CAstell, M., "Internet, libertad, y sociedad. Una perspectiva analítica", Polis, 2003, disponible en http:// redalic.org/articulo.oa?

DíAZ, Viviana Laura, Teletrabajo y neurotecnología. Una guía imprescindible para gestionar el trabajo, Buenos Aires, Ediciones Granica, 2018.

Garza Rosa, Susana, Aprender cómo aprender, México, Trillas, 1998.

GARZÓn, R. y GómeZ, N., Alfabetización laboral, México, Plaza y Valdés, 2018.

HERNÁNDEZ-SERRANO, M. J., Estrategia de búsqueda de información para la generación de conocimiento, Red de Universidad de Salamanca, 2009.

Herrscher, E., Negocios, sociedad, trabajo y valores, Buenos Aires, 2013.

Kurczyn Villalobos, Patricia et al., Derecho laboral globalizado, México, UNAM, Instituto de Investigaciones Jurídicas, 2007. 
LÓPEZ-LÓPEZ, Pedro et al., Inclusión digital: un nuevo derecho bumano, Madrid, Educación y Biblioteca, 2009.

Lessa Kerstenetzy, Celia, El Estado de bienestar social en la edad de la razón, México, Fondo de Cultura Económica, 2017.

LozANO CHAIREZ, Miguel, Sombreros blancos, tres hackers programan un mundo mejor, México, Universidad Autónoma de Baja California, 2017.

Messenger, J. C. y Gschwind, L., "Telework and the Effects on Working", Time Life, Ginebra, OIT, 2015.

NickuIN, J. M., Telecommuting: ¿What? Whit? When? and How, Singanpur, Springer Science Media, 2016.

SIERRA BENÍTEZ, Esperanza, El contenido de la relación laboral en el teletrabajo, Sevilla, Consejo Económico y Social de Andalucía, 2011.

SuÁrez BARrios, Astrid Sofía, Debates, UNAD, vol. 7, enero-diciembre de 2016.

Spitzer, Manfred, Demencia digital, México, Penguin Randow House, 2018.

Reynoso Castillo, Carlos, Los contratos de trabajo, México, UAM Azcapotzalco, 2011.

RubBini, Nora Inés y MANCUSO, G., ¿El teletrabajo necesita de una nueva metáfora?, VI Jornadas de Sociología de la UNLP, La Plata, Universidad Nacional de La Plata, Facultad de Humanidades y Ciencias de la Educación, 2010.

TOKMAN, Víctor, "Políticas de empleo en la nueva era económica", Economía, vol. XXIII, núm. 46, Lima, 2000.

Yuval, Noah Harari, 21 lecciones para el siglo XXI, México, Debate, 2018.

\section{Electrónicas}

TESAURO OIT, http://www.ilo.org/public/libdoc/ILOThesaurus/spanish/. http:// www.conectas.org/ revista-sur/conectasfundacaocarloschagasdivulgamselecionadospara-o-programa-de-incentivo-aproducao-academica-em-direitoshumanos?pg. $=2$. bttps:// mww.citrix.com/esmx/glossary/whatdigitalworkspace.html\#: : text $=U n \% 20$ espacio\%20de\%20trabajo\%20digital.

bttp:/ / www.conectas.org/ revistasur/ conectafundacaocarloschagasdivulgamselecionadospara-o-programa-de-incentivo-a-producao-academica-em-direitoshumanos?pg. $=2$. 